1996

\title{
An infrared absorption investigation of hydrogen, deuterium, and nitrogen in ZnSe grown by molecular beam epitaxy
}

Zhonghai Yu

S. L. Buczkowski

L. S. Hirsch

T. H. Myers

Follow this and additional works at: https://researchrepository.wvu.edu/faculty_publications

\section{Digital Commons Citation}

Yu, Zhonghai; Buczkowski, S. L.; Hirsch, L. S.; and Myers, T. H., "An infrared absorption investigation of hydrogen, deuterium, and nitrogen in ZnSe grown by molecular beam epitaxy" (1996). Faculty Scholarship. 50.

https://researchrepository.wvu.edu/faculty_publications/50 

in $\mathrm{ZnSe}$ grown by molecular beam epitaxy

\author{
Zhonghai Yu, ${ }^{\text {a) }}$ S. L. Buczkowski, L. S. Hirsch, and T. H. Myers ${ }^{\text {) }}$ \\ Department of Physics, West Virginia University, Morgantown, West Virginia 26506-6315
}

(Received 31 May 1996; accepted for publication 22 August 1996)

Surprising concentrations of hydrogen and deuterium, as high as $5 \times 10^{20} \mathrm{~cm}^{-3}$, were incorporated into nitrogen-doped $\mathrm{ZnSe}$ grown on GaAs by molecular beam epitaxy. Infrared absorption bands due to local vibration modes were observed at 3193 and $783 \mathrm{~cm}^{-1}$ for $\mathrm{ZnSe:N,H}$ samples, and at $2368 \mathrm{~cm}^{-1}$ for $\mathrm{ZnSe}$ :N,D samples using Fourier transform infrared spectroscopy. The isotopic shift in the absorption band agrees with predictions of a simple harmonic oscillator approximation for $\mathrm{N}-\mathrm{H}$ bonding. The variation of the absorption band associated with substitutional nitrogen with nitrogen concentration indicates that not all nitrogen is substitutional, and also exhibited significant changes related to hydrogen incorporation. (C) 1996 American Institute of Physics.

[S0021-8979(96)01423-5]

\section{INTRODUCTION}

It is well known that hydrogen passivates dopants in semiconductors by bonding to the dopant atoms to form complexes or compensating centers. Extensive studies have been made in Si and III-V materials such as GaAs and InP. ${ }^{1}$ Hydrogen compensation is a significant problem with nitrogen doping of $\mathrm{ZnSe}$ grown by metalorganic chemical vapor deposition (MOCVD) ${ }^{2}$ and gas-source molecular beam epitaxy (GSMBE). ${ }^{3}$ Absorption bands due to local vibration modes (LVMs) observed in nitrogen-doped ZnSe grown by MOCVD $^{2,4}$ are attributed to $\mathrm{N}-\mathrm{H}$ bonding. These results suggest that hydrogen forms a compensating complex with nitrogen in nitrogen-doped $\mathrm{ZnSe}$, with the hydrogen bonding primarily to the nitrogen. We report the effects of intentional incorporation of hydrogen and deuterium in nitrogen-doped $\mathrm{ZnSe}$ grown by conventional molecular beam epitaxy (MBE), and for the first time, the LVM for N-D bonding in $\mathrm{ZnSe}$. The agreement between the observed isotope shift in the LVM frequency and predictions with simple harmonic oscillator theory provides strong support for $\mathrm{N}-\mathrm{H}$ bonding. We also report the first observation of the absorption band associated with substitutional nitrogen ${ }^{5}$ in as-grown $\mathrm{ZnSe:N}$.

\section{EXPERIMENT}

Nitrogen-doped $\mathrm{ZnSe}$ layers were grown on semiinsulating, (100)-oriented GaAs substrates using a custom MBE system at West Virginia University. High purity $(7 \mathrm{~N})$ elemental zinc and selenium were used as source materials. Nitrogen doping was achieved using an rf plasma atomic nitrogen source from Oxford (Model No. CARS25) operating at plasma power of $200 \mathrm{~W}$ and a nominal system pressure $2 \times 10^{-6}$ Torr. An EPI thermal cracker using either hydrogen or deuterium gas at $1 \times 10^{-6}$ Torr system pressure was employed for hydrogen or deuterium doping. All doped samples were grown with a $0.25-\mu \mathrm{m}$-thick, undoped $\mathrm{ZnSe}$ buffer layer prior to the doped layer growth. Typical doped

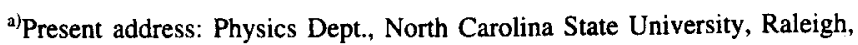
NC 27695.

b)Electronic mail: thmyers@wvnvms.wvnet.edu
}

layer thickness was $1-2 \mu \mathrm{m}$ with a growth rate of $0.2-0.4$ $\mu \mathrm{m} / \mathrm{h}$. The II/VI beam equivalent pressure (BEP) ratio ranged from 2 to 0.5 , while growth temperatures were $250^{\circ} \mathrm{C}$ and $300^{\circ} \mathrm{C}$. Substrate preparation was the same as previously reported. ${ }^{6}$

Secondary ion mass spectrometry (SIMS) measurements were made at Charles Evans and Associates (Redwood, CA) to determine the doping concentration profiles of nitrogen, hydrogen, and deuterium in $\mathrm{ZnSe}$ samples using $\mathrm{Cs}(+)$ ions. Nitrogen concentrations were determined to an absolute accuracy of $\pm 20 \%$ by direct comparison with a nitrogenimplanted $\mathrm{ZnSe}$ sample measured in the same SIMS sample set. Hydrogen and deuterium measurements were made using archived relative sensitivity factors and are quoted to within a factor of 2 . The minimum detectable concentrations of the SIMS measurement were limited by residual background gases in the SIMS system itself. Measurements made on undoped $\mathrm{ZnSe}$ layers indicated minimum detectabilities of $1 \times 10^{17}, 2 \times 10^{18}$, and $5 \times 10^{16} \mathrm{~cm}^{-3}$ for nitrogen, hydrogen, and deuterium, respectively.

\section{RESULTS AND DISCUSSION}

The depth profile for nitrogen and hydrogen concentrations in a nitrogen-doped $\mathrm{ZnSe}$ sample grown under an atomic hydrogen flux is shown in Fig. 1(a). The SIMS spectra is divided into five separate regions (dashed lines). An undoped $(u) \mathrm{ZnSe}$ buffer layer was grown directly on the GaAs substrate $(s)$. The doped portion of the layer was grown under a continuous atomic hydrogen $(a-\mathrm{H})$ flux. The rf-plasma source was used to provide atomic nitrogen $(a-\mathrm{N})$ during doped layer growth, resulting in a nitrogen concentration of about $3 \times 10^{19} \mathrm{~cm}^{-3}$ in this layer. Surprisingly, the hydrogen level was $5 \times 10^{20} \mathrm{~cm}^{-3}$ in the doped layer, which represents a concentration of almost 1 at. \%. During growth of the doped layer, the plasma was turned off while maintaining the nitrogen flow, resulting in a region grown under a molecular, rather than atomic, nitrogen flux $(m-\mathrm{N})$. As expected, there is a corresponding decrease in nitrogen concentration. The nitrogen levels then increased back to their previous values when the rf-plasma source was restarted. The 


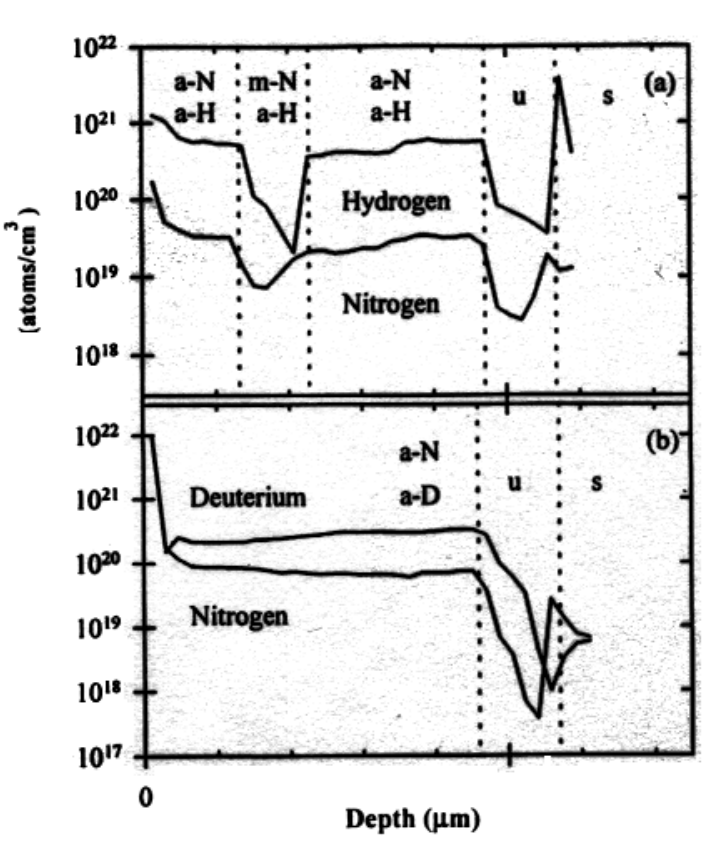

FIG. 1. SIMS profile of $\mathrm{ZnSe:N}$ layers grown by MBE (a) under an atomic hydrogen flux, and (b) under an atomic deuterium flux. Dashed lines separate regions with differing growth conditions represented by $s$ : GaAs substrate; $u$ : undoped $\mathrm{ZnSe}$ buffer layer, $a-\mathrm{H}(a-\mathrm{D})$ ): atomic hydrogen (deuterium) flux present; $a-\mathrm{N}$ : atomic nitrogen flux present; and $m-\mathrm{N}$ : molecular nitrogen flux present.

variation in hydrogen concentration throughout the sample's depth closely tracks that of nitrogen. The hydrogen incorporation was clearly influenced by nitrogen incorporation in the $\mathrm{ZnSe}$ similar to the phenomenon observed in nitrogen-doped ZnSe grown by GSMBE. ${ }^{3}$ For several layers, deuterium was used during nitrogen-doped $\mathrm{ZnSe}$ growth to investigate both the nature of the $\mathrm{N}-\mathrm{H}$ bond through isotopic substitution and low-level incorporation of hydrogen. Figure 1(b) shows the SIMS depth profile of a nitrogen-doped $\mathrm{ZnSe}$ sample grown under a deuterium flux, with the different regions labeled similar to Fig. 1(a). The nitrogen concentration was $8 \times 10^{19}$ $\mathrm{cm}^{-3}$ and the deuterium concentration was $3 \times 10^{20} \mathrm{~cm}^{-3}$, again at a surprisingly high level in the doped layer.

The abrupt spikes at the surface and the substrateepilayer interface in the SIMS data shown in Figs. 1(a) and (b) are artifacts of the measurement and contain no useful information. However, the less-than-abrupt profiles observed for nitrogen and hydrogen (deuterium) in the portion of the layer in Fig. 1(a) grown under molecular nitrogen, and in the undoped region of both layers shown in Figs. 1(a) and 1(b), are suggestive of significant diffusion from the heavily doped regions. It is not clear at this point if this is a true phenomenon since special care must be taken in sputtering rates and profiles during SIMS to get an accurate representation of concentration profiles. A more detailed study of nitrogen and hydrogen diffusion is planned for the future to address this question.

Table I contains a summary of hydrogen, deuterium and nitrogen concentrations for various growth conditions. Hydrogen incorporation in $\mathrm{ZnSe}$ is significant only when both atomic hydrogen and atomic nitrogen were present during the growth $(\mathrm{ZnSe}: \mathrm{N}, \mathrm{H})$. The decrease in hydrogen concen-
TABLE I. Hydrogen, deuterium, and nitrogen concentrations in $\mathrm{ZnSe}$ for various growth conditions. The nitrogen source if power was $200 \mathrm{~W}$ and the hydrogen/deuterium pressures were maintained at $1 \times 10^{-6}$ Torr whenever present.

\begin{tabular}{cccccc}
\hline \hline Sample & $\begin{array}{c}T_{\text {growth }} \\
\left({ }^{\circ} \mathrm{C}\right)\end{array}$ & $\begin{array}{c}\mathrm{Zn} / \mathrm{Se} \\
\text { ratio }\end{array}$ & $\begin{array}{c}\mathrm{N} \text { flux } \\
\left(\times 10^{-6} \mathrm{~T} \text { orr }\right. \\
\mathrm{BEP})\end{array}$ & $\begin{array}{c}{[\mathrm{N}]} \\
\left(\times 10^{19} \mathrm{~cm}^{-3}\right)\end{array}$ & $\begin{array}{c}{[\mathrm{H}] \text { or }[\mathrm{D}]} \\
\left(\times 10^{19} \mathrm{~cm}^{-3}\right)\end{array}$ \\
\hline & 250 & 1.8 & 3.0 & 3.0 & 50 \\
& 250 & 2.0 & 4.7 & 8.0 & 30 \\
& 250 & 0.6 & 4.7 & 1.5 & 1.8 \\
& 300 & 0.5 & 1.5 & 0.4 & 1.0 \\
& 300 & 0.5 & 3.2 & 1.5 & background \\
& 300 & 0.5 & 0 & background & background \\
& 300 & 1.2 & 5.1 & 8.0 & 0.1 \\
\hline
\end{tabular}

tration when molecular nitrogen replaces atomic nitrogen during growth shown in Fig. 1(a) clearly demonstrates this effect. In nitrogen-doped $\mathrm{ZnSe}$ exposed to molecular deuterium during growth (ZnSe:N, $\left.\mathrm{D}_{2}\right)$, a deuterium level at $10^{18}$ $\mathrm{cm}^{-3}$ and nitrogen level at $8 \times 10^{19} \mathrm{~cm}^{-3}$ were observed, indicating a low hydrogen incorporation efficiency for molecular vs atomic deuterium. This low "cracking" efficiency indicates that the background levels of molecular hydrogen typically found in an MBE system may not be an issue for compensation of nitrogen-doped $\mathrm{ZnSe}$. In addition, we did not observe measurable deuterium incorporation for undoped layers grown under an atomic deuterium flux. Hydrogen incorporation in $\mathrm{ZnSe}$ also depends on the growth conditions. The highest nitrogen and hydrogen concentrations were obtained for $\mathrm{Zn}$-rich conditions at the lower growth temperature. The highest hydrogen level was $5 \times 10^{20} \mathrm{~cm}^{-3}$, more than one order higher than the nitrogen level in the same layer. As expected, $\mathrm{Zn}$-rich conditions are more favorable to nitrogen incorporation in $\mathrm{ZnSe}$ since nitrogen occupies a $\mathrm{Se}$ site for $p$-type doping in $\mathrm{ZnSe}$. $^{7,8}$

Fourier transform infrared (FTIR) spectroscopy was performed both at room temperature and $15 \mathrm{~K}$ on the $\mathrm{ZnSe}$ samples using a Nicolet model 550 FTIR spectrometer. Absorption bands were observed at 783 and $3193 \mathrm{~cm}^{-1}$ in nitrogen-doped $\mathrm{ZnSe}$ samples grown under an atomic hydrogen flux similar to that previously reported for MOCVD growth. ${ }^{2,4}$ These peaks were absent from $\mathrm{ZnSe}$ samples grown only with either an atomic nitrogen or an atomic hydrogen flux, as well as from undoped $\mathrm{ZnSe}$ samples. The $3194 \mathrm{~cm}^{-1}$ band has been tentatively assigned to the stretching mode, and the $783 \mathrm{~cm}^{-1}$ band to the wagging mode of the $\mathrm{N}-\mathrm{H}$ bond in $\mathrm{ZnSe}$ based on absorption bands observed in ammonia ${ }^{1}$ and $\mathrm{N}-\mathrm{H}$ bonding in $\mathrm{GaAs}$ and $\mathrm{GaP} .{ }^{9}$

The effect of isotopic substitution on the observed vibrational frequency is very useful for identification of the species that comprise a defect complex. ${ }^{1,10}$ For example, the large frequency shift that results upon the substitution of deuterium for hydrogen leads to an unambiguous assignment of hydrogen bonding. In the harmonic oscillator approximation, the vibrational frequency of an $\mathrm{X}-\mathrm{H}$ oscillator is assumed to be inversely proportional to the square root of the reduced mass of the oscillator, $\omega(\mathrm{H}) \propto\left[m_{\mathrm{X}} m_{\mathrm{H}} /\left(m_{\mathrm{X}}+m_{\mathrm{H}}\right)\right]^{-1 / 2}$, where $m_{\mathrm{H}}$ and $m_{\mathrm{X}}$ are the masses of hydrogen and the other 


\section{tice relaxation related to $\mathrm{N}-\mathrm{H}$ bond formation.}

Comparison of Figs. 3(a) and 3(b) indicate that the absorption band at $553 \mathrm{~cm}^{-1}$ increases with increasing nitrogen concentration. However, the relative increase in area under the absorption curve does not increase proportionately to the increase in nitrogen concentration. Based on the integrated area under the peak shown as Fig. 3(b), we would have expected the absorption peak shown in Fig. 3(a) to be approximately twice as large. Though preliminary, this result is direct evidence that a significant proportion of the incorporated nitrogen does not occupy a simple substitutional site. Thus, there are at least two inequivalent sites for nitrogen incorporation. Since these concentrations are above the theoretical solubility limit for substitutional $\mathrm{N}$ in $\mathrm{ZnSe},{ }^{15}$ this may provide indirect evidence of $\mathrm{N}-\mathrm{N}$ pairing. It is well known that a self-compensation mechanism exists for nitrogen doping of $\mathrm{ZnSe}$ based on transport measurements, ${ }^{16-18}$ and that significant non-radiative centers are created above a certain nitrogen concentration level. ${ }^{19,20}$ At present, however, the actual mechanisms for compensation are still speculative. ${ }^{21}$ Further study of the relationship between FTIR absorption signatures and $\mathrm{N}$ concentration is planned, since understanding of this phenomenon may help us to identify the mechanisms responsible for limiting the maximum level of $p$-type doping in ZnSe.

\section{SUMMARY}

In conclusion, hydrogen and deuterium were incorporated into nitrogen-doped ZnSe grown by MBE. Surprisingly high levels of hydrogen and deuterium were observed, with hydrogen concentrations as high as $5 \times 10^{20} \mathrm{~cm}^{-3}$ in a $\mathrm{ZnSe}: \mathrm{N}$ layer with a nitrogen concentration at $3 \times 10^{19} \mathrm{~cm}^{-3}$. It was found that hydrogen incorporation closely tracks the nitrogen incorporation in $\mathrm{ZnSe}$. The hydrogen incorporation was significant only when both atomic hydrogen and atomic nitrogen were present during growth. Samples with the highest hydrogen and nitrogen concentrations were grown under a $\mathrm{Zn}$-rich condition. Local vibration modes were observed at 3193 and $783 \mathrm{~cm}^{-1}$ for $\mathrm{ZnSe}: \mathrm{N}, \mathrm{H}$ samples and at $2368 \mathrm{~cm}^{-1}$ for $\mathrm{ZnSe:N,D} \mathrm{samples} \mathrm{by} \mathrm{Fourier} \mathrm{transform} \mathrm{infrared} \mathrm{spec-}$ troscopy. The isotopic LVM peak shift agrees very well with the prediction from a one-dimensional harmonic oscillator approximation which strongly supports the formation of $\mathrm{N}-\mathrm{H}$ bonds in $\mathrm{ZnSe}$. Observations of the variation of the 553 $\mathrm{cm}^{-1}$ absorption band associated with substitutional nitrogen are consistent with $\mathrm{N}-\mathrm{H}$ bonding and provide evidence of nonsubstitutional nitrogen incorporation possibly related to $\mathrm{N}-\mathrm{N}$ pairing in heavily doped samples.

This work was supported by the West Virginia/National Science Foundation EPSCoR program and two NSF instrumentation Grants, Nos. DMR92-08130 and DMR92-14350. We would like to extend a special appreciation to L. E. Halliburton for sharing the FTIR facilities.

${ }^{1}$ See, for example, in Semiconductors and Semimetals: Hydrogen in Semiconductors, edited by J. I. Pankove and N. M. Johnson (Academic, New York, 1991), Vol. 34.

${ }^{2}$ A. Kamata, H. Mitsuhashi, and H. Fujita, Appl. Phys. Lett. 63, 3353 (1993).

${ }^{3}$ E. Ho, P. A. Fisher, J. L. House, G. S. Petrich, L. A. Kolodziejski, J. Walker, and N. M. Johnson, Appl. Phys. Lett. 66, 1062 (1995).

${ }^{4}$ J. A. Work, J. W. Ager III, K. J. Duxstad, E. E. Haller, N. R. Taskar, D. R. Dorman, and D. J. Olego, Appl. Phys. Lett. 63, 2756 (1993).

${ }^{5}$ H. J. Stein, Appl. Phys. Lett. 64, 1520 (1994).

${ }^{6}$ Zhonghai Yu, S. L. Buczkowski, N. C. Giles, and T. H. Myers, Appl. Phys. Lett. 69, 82 (1996).

${ }^{7}$ Y. Kawakami, T. Ohnakado, M. Tsuka, S. Tokudera, Y. Ito, S. Fujita, and S. Fujita, J. Vac. Sci. Technol. B 11, 2057 (1993).

${ }^{8}$ S. Matsumoto, H. Tosaka, T. Yoshida, M. Kobayashi, and A. Yoshikawa, Jpn. J. Appl. Phys. 32, L229 (1993).

${ }^{9}$ See Ref. 4 and the references therein.

${ }^{10}$ B. Pajot, B. Clerjaud, and J. Chevallier, Physica B 170, 371 (1991).

"I. Neugebauer and C. G. Van de Walle, Appl. Phys. Lett. 68, 1829 (1996)

${ }^{12}$ Z. Yu, S. L. Buczkowski, M. C. Petcu, N. C. Giles, and T. H. Myers, Appl. Phys. Lett. 68, 529 (1996).

${ }^{13}$ G. G. Deleo, Physica B 170, 295 (1991).

${ }^{14} \mathrm{~J}$. Tatarkiewicz, A. Breitschwerdt, A. M. Witowski, and H. Hartmann, Solid State Commun. 68, 1081 (1988).

${ }^{15}$ D. B. Laks, C. G. Van de Walle, G. F. Neumark, and S. T. Pantelides, Appl. Phys. Lett. 63, 1375 (1993).

${ }^{16}$ J. Qui, J. M. DePuydt, H. Cheng, and M. A. Haase, Appl. Phys. Lett. 59. 2992 (1991).

${ }^{17}$ E. Kurtz, S. Einfeldt, J. Nurnberger, S. Zerlauth, D. Hommel, and G. Landwehr, Phys. Status Solidi B 187, 393 (1995).

${ }^{18}$ K. A. Prior. Phys. Status Solidi B 187, 379 (1995).

${ }^{19}$ L. C. Calhoun, C. M. Rouleau, M. H. Jeon, and R. M. Park, J. Cryst. Growth 138, 352 (1994).

${ }^{20}$ J. W. Allen, D. T. Reid, W. Sibbett, W. Sleat, J.-Z. Zheng, D. Hommel, and B. Jobst, J. Appl. Phys. 78, 1731 (1995).

${ }^{21}$ C. H. Park and D. J. Chadi, Phys. Rev. Lett. 75, 1134 (1995). 